\title{
TIME-DEPENDENT SURFACE ELEVATION OF AN ICE SLOPE*
}

\author{
By Kolumban Hutter \\ (Versuchsanstalt für Wasserbau, Hydrologie und Glaziologie, Eidg. Technische Hochschule \\ Zürich, Gloriastrasse 37/39, CH-8og2 Zürich, Switzerland)
}

\begin{abstract}
By introducing a coordinate stretching, the governing field equations of the creep flow of a non-Newtonian viscous medium down a uniform slope are solved to determine the differential equation describing the propagation of long surface waves caused by initial disturbances and/or time-dependent accumulation-rate. The differential equation for the surface wave depends on the flow law of the nonNewtonian fluid, the boundary condition at the ice-bedrock interface, the bedrock topography, and the thickness-wavelength ratio. For moderately long waves and small elevation above the mean thickness the results agree in their essentials with those of the kinematic wave theory and the forward wave equation with a diffusion term is derived, but when improving this by allowing higher elevations the Burger's equation and even more complex equations are obtained. To derive these results Glen's flow law must be generalized to avoid infinitely fast changes in stress deviators close to zero strain-rates. The range of applicability of the various equations is discussed.
\end{abstract}

RÉsumé. Altitude en fonction du temps d'une pente de glace. En introduisant une coordonnée de tension, on a résolu les équations qui régissent le glissement d'un milieu visqueux non-Newtonien le long d'une pente uniforme afin de déterminer l'équation différentielle de la propagation des longues ondulations de surface causées par les irrégularités initiales et/ou le taux d'accumulation en fonction du temps. L'équation différentielle pour l'ondulation de surface dépend de la loi d'écoulement du fluide non-Newtonien, des conditions aux limites à l'interface glace-lit rocheux, de la topographie du lit rocheux et du rapport entre la longueur d'onde de l'ondulation et l'épaisseur. Pour des ondulations modérément longues et une faible dénivellée au-dessus de l'épaisseur moyenne, les résultats concordent dans l'ensemble avec ceux de la théorie des ondes cinématiques et on en déduit l'équation de la propagation de l'onde avec un terme de diffusion, mais si on veut poursuivre en admettant des dénivellées plus importantes, on obtient l'équation de Burger et même des équations plus complexes. Pour retrouver ces résultats, il faut généraliser les lois d'écoulement de Glen de manière à éviter des variations infiniment rapides des déviateurs des contraintes au voisinage des taux de déformation nuls. On discute des domaines d'applicabilité des différentes équations.

Zusammenfassung. Zeitabhängige Oberflächenerhebungen in einer Eisschicht. Durch Einführung einer Koordinatenstreckung werden die Feldgleichungen der Kriechbewegung eines nicht-Newtonschen Mediums für eine planparallele Schicht gelöst; dadurch erhält man die Differentialsgleichung für lange Oberflächenwellen, welche durch anfängliche Störungen und zeitabhängige Akkumulationsraten erzeugt werden. Die Differentialgleichung dieser Oberflächenwellen hängt vom Fliessgesetz der nicht-Newtonschen Flüssigkeit, von der Randbedingung am Gletscherbett, der Untergrundtopographie und vom Verhältnis Gletscherdicke/ Wellenlänge ab. Für mässig lange Wellen und kleine Oberflächenerhebungen stimmen die Resultate grundsätzlich mit der Theorie kinematischer. Wellen überein. Man erhält die Gleichung einer vorwärts schreitenden Welle mit einem zusätzlichen Diffusionsterm. Wenn die Theorie durch Mitberücksichtigung grosser Oberflächenerhebungen verbessert wird, so erhält man jedoch die Gleichung von Burgers, oder noch kompliziertere Wellengleichungen, welche die Dispersion mitberücksichtigen. Um diese Resultate herzuleiten, muss das Glen'sche Fliessgesetz verallgemeinert werden, um unendlich schnelle Änderungen im Spannungsdeviator bei kleinen Verzerrungsgeschwindigkeiten zu vermeiden. Der Berórch der Anwendbarkeit der verschiedenen Gleichungen wird diskutiert und es wird gezeigt, dass die kinematische Wellentheorie i.a. nicht genügt, um Oberflächenwellen in Gletschern zu behandeln.

\section{Introduction}

Undulations on the surface of ice sheets with a predominant wavelength of the order of several times the ice thickness have been studied by many authors in the past, notably by Budd (I970[a], [b], I97I) who also gives an account of the relevant literature. For steadystate conditions he was able to relate the frequency spectrum of the bedrock topography to that of the surface and could explain, for instance, under what circumstances the basal shear stress fluctuates in sympathy with the surface slope. Budd considered the non-Newtonian

* Dedicated to Professor Dr H. Parkus on the occasion of his seventieth birthday, 31 January 1979. 
viscous flow of a medium down a uniform slope with small harmonic undulations superimposed on it, but resorted to an approximate solution technique. A clear separation of the solution for bedrock undulations and steady-state accumulation-rate was not attempted, nor was there any systematic treatment of the transient response. Moreover, some of his boundary conditions were derived using an integrated mass-balance law, an unnecessary and doubtful procedure.

For these reasons, Hutter and others (in press) re-investigated the entire matter anew, first aiming at a clear and systematic formulation of the governing equations, secondly separating the steady-state and the transient response; and, thirdly, attempting to use a perturbation technique to answer various questions concerning the state of stress and velocity in a nearly parallel-sided slab. In this paper the analysis of Hutter and others is continued insofar as we investigate the time-dependent response. Our interest is, in particular, in the variation of the surface undulations as a result of initial disturbances and/or time-dependent accumulation rate. This must, under certain circumstances produce surface waves travelling down the glacier. Such waves were treated by Nye (196o, $\left.\mathrm{I}_{96}[\mathrm{a}],[\mathrm{b}]\right)$ using kinematic wave theory. In that theory the ice slope is regarded as a whole. Balance of mass forms the only significant field equation, and balance of momentum is virtually abandoned, except, perhaps, as it enters the continuity equation through a phenomenological equation connecting discharge with depth and surface inclination.

Contrary to kinematic wave theory, the present approach makes full use of the momentum equation, in which, however, acceleration terms are discarded. The mathematical complexities introduced thereby are overcome by a stretching of coordinates. This restricts the disturbances to surface waves with wavelengths which are long compared to the mean thickness of the ice sheet, but short compared to its length; however, the method has the advantage that it delivers the wave speeds and the diffusivities as functions of the geometry of the ice slope and the boundary conditions at the ice-bedrock interface in a much more systematic way than is possible with the kinematic wave theory. Two features are new when compared with kinematic wave theory. First, the governing equation for the surface elevation depends on the ratio of ice thickness to a typical wavelength. Secondly, Glen's flow law must be generalized, because otherwise our proposed solution technique fails. The reason for this failure is natural, because in Glen's flow law stress deviators grow infinitely fast at low strainrates, a singular behaviour that leads to singular integrals in the solutions.

\section{Governing equations}

Consider slow flow of a viscous medium of uniform thickness down a uniform slope. Let $(x, y)$ be a Cartesian coordinate system; $x$ is down and $y$ normal to the plane. Further, let $(u, v)$ be the components of the velocity vector $\mathbf{v}$ in the $x$ - and $y$-directions respectively, and denote by $\mathbf{D}=\frac{1}{2}(\nabla \mathbf{v}+\mathbf{v} \nabla)$ the stretching tensor. The governing equations for the twodimensional motion of a medium are the balance of mass and momentum and the constitutive relationship for stress. For a broad class of non-Newtonian incompressible fluids the latter may be written as

$$
D_{i j}=A B\left(t_{\mathrm{II}}{ }^{\prime 2}\right) t_{i j}{ }^{\prime}, \quad t_{\mathrm{II}}{ }^{2}=\frac{1}{2} t_{i j}{ }^{\prime} t_{i j}{ }^{\prime} .
$$

where $t_{i j}{ }^{\prime}$ is the stress deviator (the stress tensor will be denoted by $t_{i j}$ ) and $t_{\mathrm{II}^{\prime 2}}$ its second invariant, $A$ is temperature dependent in general, but will henceforth be assumed constant; this is only a qualitative limitation which does not affect the essential conclusions of the calculations. $B$ is an as yet unspecified function; for Glen's flow law $B(x)=x^{(n-1) / 2}$.

In what follows it is advantageous to non-dimensionalize lengths, stresses, and time with the mean thickness of the undisturbed, strictly parallel-sided slab, $D, \sigma_{0}=\rho g D$ where $\rho$ is the ice density and $g$ the gravitational constant, and $\tau=A^{-1}\left[\rho g D B\left(\rho^{2} g^{2} D^{2}\right)\right]^{-1}$. Thus 


$$
\begin{aligned}
(x, y)_{\mathrm{dim}} & =D(x, y), \\
\left(t_{x x}, t_{y y}, t_{x y}\right) & =\sigma_{0}\left(\sigma_{x}, \sigma_{y}, \tau\right), \\
(u, v)_{\operatorname{dim}} & =\frac{D}{\tau}(u, v), \\
t_{\mathrm{dim}} & =\tau t .
\end{aligned}
$$

For ice and Glen's flow law $\tau$ is in the order of $\mathrm{I}$ to $3 \mathrm{~d}^{*}$.

In non-dimensional variables, the governing field equations are:

$$
\begin{aligned}
& \frac{\partial u}{\partial x}+\frac{\partial v}{\partial y}=0, \\
& \frac{\partial \sigma_{x}}{\partial x}+\frac{\partial \tau}{\partial y}+\sin \gamma=\mathbb{\nexists}\left\{\frac{\partial u}{\partial t}+\frac{\partial u}{\partial x} u+\frac{\partial u}{\partial y} v\right\}, \\
& \frac{\partial \tau}{\partial x}+\frac{\partial \sigma_{y}}{\partial y}-\cos \gamma=\mathbb{T}\left\{\frac{\partial v}{\partial t}+\frac{\partial v}{\partial x} u+\frac{\partial v}{\partial y} v\right\} \text {, } \\
& \frac{\partial u}{\partial x}=\frac{\mathrm{I}}{2} \boldsymbol{g}\left(\boldsymbol{\tau}_{\mathrm{II}}{ }^{2}\right)\left(\sigma_{x}-\sigma_{y}\right) \text {, } \\
& \frac{\partial u}{\partial y}+\frac{\partial v}{\partial x}=2{ }_{2}\left(\tau_{I_{I}}{ }^{\prime 2}\right) \tau .
\end{aligned}
$$

where $\gamma$ is the slope angle, and $u, v$ are the dimensionless velocity components in the $x$ and $y$ directions, respectively, and

$$
\left.\begin{array}{c}
\text { 现 }=\frac{D}{g \tau^{2}}, \\
\mathbb{Z}\left(\tau_{I^{\prime}}{ }^{\prime 2}\right)=\frac{B\left(\rho^{2} g^{2} D^{2} \tau_{I I^{\prime 2}}\right)}{B\left(\rho^{2} g^{2} D^{2}\right)}, \\
\tau_{I^{\prime}}{ }^{2}=\frac{1}{4}\left(\sigma_{x}-\sigma_{y}\right)^{2}+\tau^{2} .
\end{array}\right\}
$$

For ice obeying Glen's flow law, 7 is smaller than $10^{-7}$, for $D=1$ ooo $\mathrm{m}, \tau=10^{5} \mathrm{~s}$, 项 $=\mathrm{IO}^{-8}$. Acceleration terms may therefore safely be neglected. Equations (3) are to be solved subject to the boundary conditions:

at the base, i.e. at $y=0$,

$$
\left.\begin{array}{l}
u=\phi \tau^{m}, \\
v=0,
\end{array}\right\}
$$

in which $\phi$ is a constant whose value may substantially vary from glacier to glacier, and at the surface, i.e. at $y=r(x, t)$,

$$
\left.\begin{array}{c}
\frac{\partial Y}{\partial t}+\frac{\partial Y}{\partial x} u-v=\mathfrak{A}, \\
\cos ^{2} \alpha+\tau \sin 2 \alpha=-p, \\
\left(\sigma_{y}-\sigma_{x}\right) \tan 2 \alpha=0 .
\end{array}\right\}
$$

* $D=10^{3} \mathrm{~m}, n=3$, and $A=5 \times 10^{-24} \mathrm{~m}^{6} / \mathrm{N}^{3} \mathrm{~s}$ yields $\tau=10^{5} \mathrm{~s}$, but glacier thicknesses vary. 
In these equations $\mathfrak{A}$ denotes the non-dimensional accumulation-rate, $\mathfrak{A}_{\text {dim }}=\mathfrak{A} D / \tau$; for real glaciers and ice sheets $\mathfrak{A}$ is smaller than $10^{-2}$ to $10^{-4}$, and thus very small; $p$ is the dimensionless atmospheric pressure and $-\alpha$ the inclination to the $x$-axis,

$$
\tan \alpha=-\frac{\partial Y}{\partial x} \text {. }
$$

The first of Equations (5) is a non-linear sliding law accounting for a possible regelation mechanism. For $\phi=0$ it includes the no-slip condition. As a first approximation we have neglected a thorough treatment of bedrock undulations. Very roughly, they are incorporated in Equations (5), however, and can be taken into account by adjusting $\phi$ accordingly.

A steady solution of Equations (3), (5), and (6), henceforth denoted by a circumflex and valid for vanishing steady accumulation rate $\mathfrak{A}=0$, is

$$
\begin{aligned}
& \hat{\tau}=\sin \gamma(\mathrm{I}-y), \\
& \hat{\sigma}_{x}=\hat{\sigma}_{y}=-\cos \gamma(\mathrm{I}-y)-p, \\
& \hat{u}=2 \int_{0}^{y} \hat{R}\left(\sin ^{2} \gamma\left(\mathrm{I}-y^{\prime}\right)^{2}\right) \sin \gamma\left(\mathrm{I}-y^{\prime}\right) \mathrm{d} y^{\prime}+\phi \sin ^{m} \gamma, \\
& \hat{v}=0, \quad \hat{r}=\mathrm{I}, \quad \hat{\alpha}=0 .
\end{aligned}
$$

Here a comment regarding the choice $\mathfrak{\mathbb { A }}=0$ is in order. In actual glaciers this condition is satisfied only at the snow-line, but not throughout the entire glacier. As a consequence the formulae of the parallel-sided slab as expressed by Equations (8) cannot form a real solution in glaciers. Steady-state solutions of real glaciers must instead lead to nearly parallel-sided slabs with slowly varying top surfaces. The subsequent analysis could indeed be based on such a weaker postulate, but calculations would become unwieldy and transparancy in the physical interpretation would probably be lost. (Incidentally, I use this more general approach in an article dealing with the influence of longitudinal strain on basal shear, see Hutter (in press).) The restriction to the case $\mathfrak{A}=0$ is not a serious drawback, however, as the major conclusions do not depend on it.

Consider fluctuations on the motion given by Equations (8); denote these by tildes so that $u=\hat{u}+\tilde{u}$. The governing equations can now be separated for the two parts. We shall perform this separation under the assumption that the steady-state stresses are large in comparison to the stresses set up by the transient motion. The perturbation equations can then be linearized with respect to the stresses so that the following perturbation equations are obtained:

$$
\begin{aligned}
& \frac{\partial \tilde{\sigma}_{x}}{\partial x}+\frac{\partial \tilde{\tau}}{\partial y}=\mathrm{o}, \\
& \frac{\partial \tilde{\tau}}{\partial x}+\frac{\partial \tilde{\sigma}_{y}}{\partial y}=\mathrm{o}, \\
& \frac{\partial \tilde{u}}{\partial x}+\frac{\partial \tilde{v}}{\partial y}=o,
\end{aligned}
$$

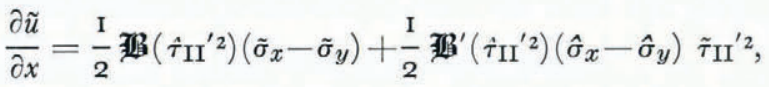

$$
\begin{aligned}
& \frac{\partial \tilde{u}}{\partial y}+\frac{\partial \tilde{v}}{\partial x}=2 \mathbb{Z}\left(\hat{\tau}_{\mathrm{II}^{\prime}}{ }^{2}\right) \tilde{\tau}+2 \mathbf{X}^{\prime}\left(\hat{\tau}_{\mathrm{II}^{\prime 2}}\right) \hat{\tau} \tilde{\tau}_{\mathrm{II}}{ }^{\prime 2} \text {, }
\end{aligned}
$$


in which

and

$$
\left.\begin{array}{rl}
\hat{\tau}_{\mathrm{II}}{ }^{\prime 2} & =\frac{1}{4}\left(\hat{\sigma}_{x}-\hat{\sigma}_{y}\right)^{2}+\hat{\tau}^{2}, \\
\hat{\tau}_{\mathrm{II}}{ }^{\prime 2} & =2\left[\frac{1}{4}\left(\hat{\sigma}_{x}-\hat{\sigma}_{y}\right)\left(\tilde{\sigma}_{x}-\tilde{\sigma}_{y}\right)+\hat{\tau} \tilde{\tau}\right], \\
\boldsymbol{g}^{\prime}(x) & =\mathrm{d} \mathfrak{g}(x) / \mathrm{d} x .
\end{array}\right\}
$$

In this paper we shall consider long surface waves; in other words, wavelengths of the time-dependent surface undulations will be assumed to be large in comparison to the mean glacier thickness. Needless to say the total length of the glacier is also assumed to be large compared to these wavelengths. Based on this we introduce the stretchings

$$
\left.\begin{array}{r}
x \rightarrow \xi / \mu, \quad y \rightarrow \eta, \quad t \rightarrow \tau / \mu, \\
\tilde{u} \rightarrow \tilde{u}, \quad \tilde{v} \rightarrow \mu \tilde{v},
\end{array}\right\}
$$

where $\mu$ may be interpreted as the ratio of the mean thickness to a characteristic wavelength. Incorporating Equations (I I) in Equations (9) gives (deleting tildes)

$$
\begin{aligned}
& \mu \frac{\partial \sigma_{x}}{\partial \xi}+\frac{\partial \tau}{\partial \eta}=0 \\
& \mu \frac{\partial \tau}{\partial \xi}+\frac{\partial \sigma_{y}}{\partial \eta}=\mathbf{o} \\
& \frac{\partial u}{\partial \xi}+\frac{\partial v}{\partial \eta}=o,
\end{aligned}
$$

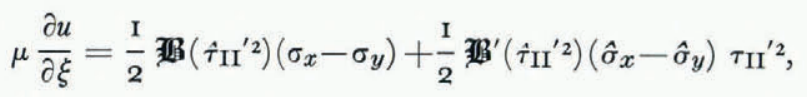

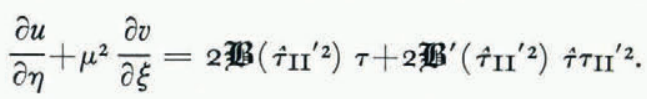

In these equations quantities with a circumflex are known from the solution of the steady-state problem, and $\hat{\tau}_{\mathrm{II}}{ }^{2}$ is given in Equations (I0). Equations (I2) must be solved subject to the boundary conditions, at the base $(y=0)$

at the top surface $(y=\Upsilon(\xi, \tau))$

$$
\left.\begin{array}{l}
u=m \phi \hat{\tau}^{m-1} \tau, \\
v=0,
\end{array}\right\}
$$

$$
\left.\begin{array}{rl}
\frac{\partial r}{\partial \tau}+(\hat{u}+u) \frac{\partial Y}{\partial \xi}-v & =\frac{\mathfrak{A}}{\mu}, \\
\sigma_{y}+\hat{\sigma}_{y}-2 \mu(\tau+\hat{\tau}) \frac{\partial r}{\partial \xi}+\mu^{2}\left(\sigma_{x}+\hat{\sigma}_{x}-\sigma_{y}-\hat{\sigma}_{y}\right)\left(\frac{\partial r}{\partial \xi}\right)^{2} & =-p, \\
\tau-\mu\left(\sigma_{x}-\sigma_{y}\right) \frac{\partial r}{\partial \xi}+\hat{\tau}+O\left(\mu^{3}\right) & =0 .
\end{array}\right\}
$$

It is about at this point that we should comment on the introduction of the stretchings, Equations (I I). Time was stretched to balance all members on the left-hand side of the first of Equations (14). Only in this case does it admit possible wave-like solutions. We shall see corroboration for this in the following section, because the emerging equation for surface 
elevation will essentially be a forward wave equation. The stretchings were further such that the kinematic boundary condition in Equations (14) was preserved; this was not so with all other stretchings we tried. Incidentally it is well known in fluid mechanics (see, e.g. Stoker, 1957; Benney, 1966) and may, for instance, be used to derive the shallow-water equations. But what have we gained? The stretching parameter has explicitly entered the governing equations, and since it is small this suggests a perturbation solution technique which carries the advantage that surface-wave and velocity field have been mathematically decoupled: the solution of the former can be used in the determination of the latter. This will become clear below.

Our aim is to develop asymptotic solutions of the non-linear problem for $\mu$ small. The physical interest is in waves induced by the accumulation/ablation rate $\mathfrak{A}$.

We emphasize that Equations (12)-(14) are for long waves of small amplitudes even though they are formally written for finite amplitudes. Solutions must therefore be sought in the form

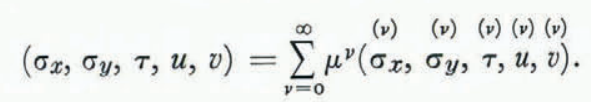

These will be restricted to the steady-state results of Equations (8), which themselves are based on Equations (5) that neglect bedrock topography. Small undulations of the latter could be introduced by means of a second perturbation parameter. This will not be attempted in this paper.

\section{On Glen's flow LAW}

Despite its general acceptance in glaciology, Glen's flow law has its shortcomings. The most serious one is its singular behaviour at small stress deviators and strain-rates: for very small strain-rates the stresses grow infinitely fast. To avoid such pathological behaviour Glen's flow law can, for instance, be altered by introducing a polynomial law instead. This was suggested by Lliboutry (1969). By simply fitting experimental data he obtained

$$
B(x)=a+b x^{\frac{1}{2}}+c x,
$$

where $a, b$, and $c$ are temperature dependent at most. Unlike Glen's law, $B(x)=X^{(n-1) / 2}$, the constitutive relationship for the stress deviator based on Equation (I6) no longer shows the singular behaviour at zero stresses.

There have been other proposals to change Glen's flow law. Barnes and others (I97I) and Ramseier and Dickins (1972), for instance, suggest instead of Equation (16) a hyperbolic sine

$$
B(x)=\sinh ^{n}\left(\beta x^{\frac{1}{2}}\right) / x,
$$

where $n$ and $\beta$ are constants, but such a law still leads to infinite viscosities and thus does not remove the mathematical singularities mentioned above. For that purpose a finite-viscosity law must be developed. Colbeck and Evans (1973) give a form similar to that of Lliboutry. On the other hand, using a model of statistical mechanics, in which rate-process theory is used, the law

$$
B(x)=\sinh (\mathrm{f}(\sqrt{ } x))
$$

where $f(\cdot)$ is a polynomial, can be derived (a paper on this is in preparation). A power-series expansion for small $x$ reduces this to a polynomial similar to Equation (15).

For most computational purposes the odd behaviour of Glen's law does not matter. This paper is an exception, because the mathematical approach used here will become singular when based on a Glen-type power law. In order to avoid unnecessary complications, we shall, henceforth, use the simplest extension avoiding this singularity, namely

$$
B(x)=x^{(n-1) / 2}+a,
$$


so that in view of the definition of

$$
\boldsymbol{Z}(x)=\frac{x^{(n-\mathrm{I}) / 2}+\mathfrak{a}}{\mathrm{I}+\mathfrak{a}}, \quad \text { where } \mathfrak{a}=a /(\rho g D)^{n-1} .
$$

$\mathfrak{a}$ is, clearly, dimensionless, and for $\mathbf{a}=$ o Glen's flow law is obtained. A similar extension is also used by Thompson (r979).

When introducing Equation (I9) three constants, namely $A$, a, $n$ must be adjusted to the experiments. To this end, denote the numerical values of these constants in Glen's flow law by $A_{\mathrm{G}}$ and $n_{\mathrm{G}}$. Requiring agreement of Equation (I9) with Glen's law at large stresses gives

$$
A=A_{\mathrm{G}}, \quad n=n_{\mathrm{G}} .
$$

Knowing these, $a$ or a follows by matching Equation (19) as $x \rightarrow 0$ with low-stress experiments as performed, e.g. by Jellinek and Brill (1956) or Frankenstein (1968); an order of magnitude is

$$
n=2, \quad \mathfrak{a} \approx \mathrm{IO}^{-2} ; \quad n=3, \quad \mathfrak{a} \approx \mathrm{IO}^{-3} .
$$

Before we present the solution technique a warning is in order which puts the mathematical approach into the proper perspectives. It is true that the difficulty with the use of Glen's flow law is a mathematical one connected to the proposed perturbation approach. (It has also arisen in finite-difference and other solution techniques of the respective equations, see Hutter and others (1979).) As $\mathfrak{a} \rightarrow$ o the solution technique fails. It would, therefore, be appropriate to search for a mathematical procedure which remains regular as $\mathfrak{a} \rightarrow 0$. Such a technique would have to be sought using a multiple-variable expansion procedure (see Cole, I968; Nayfeh, 1973; Van Dyke, 1975). We are less ambitious here and are satisfied with a solution technique that is valid for a bounded away from zero. The risk in such a procedure is that singularities occur in higher order terms as $\mathfrak{a} \rightarrow 0$. This is true and a disadvantage, but we find support for our approach in the experimental literature mentioned above, which seems to prove that $\mathbf{a}$ is bounded away from zero.

\section{LONG WAVES ON AN INFINITE ICE SLAB}

In this section we present the method of solution for the flow of a viscous medium of uniform thickness down a uniform slope. When the perturbation expansions ( 15 ) are substituted into Equations (12)-(14) a hierarchy of initial-boundary-value problems is obtained. The zeroth-order equations are

$$
\begin{aligned}
& \frac{\partial \tau}{\partial \eta}=0, \quad \frac{\partial \sigma_{y}}{\partial \eta}=0, \quad \stackrel{(0)}{\sigma_{x}}=\stackrel{(0)}{\sigma_{y}}, \\
& \frac{\partial u}{\partial \xi}+\frac{\partial v}{\partial \eta}=0
\end{aligned}
$$

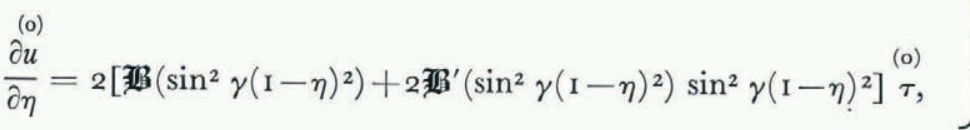

subject to the boundary conditions

$$
\stackrel{(0)}{u}=m \phi \sin ^{m-1} \gamma \tau, \quad \stackrel{(0)}{v}=0, \quad \text { at } \eta=0,
$$

* A justification for these is given in Hutter and others (in press). 
and

$$
\begin{aligned}
& \stackrel{\text { (o) }}{\sigma_{y}}=\cos \gamma(\mathrm{I}-\gamma) \text {, } \\
& \text { (o) } \\
& \tau=-\sin \gamma(\mathrm{I}-\gamma) \text {, } \\
& \frac{\partial \Upsilon}{\partial \tau}+\frac{\partial \Upsilon}{\partial \xi}\left\{2 \int_{0}^{r} \boldsymbol{z}\left(\sin ^{2} \gamma(\mathrm{I}-y)^{2}\right) \sin \gamma(\mathrm{I}-y) \mathrm{d} y\right. \\
& \left.+\phi \sin ^{m} \gamma+{ }_{u}^{(0)}\right\} \stackrel{(0)}{-v}=\frac{\mathfrak{A}}{\mu} . \\
& \text { at } \eta=\Upsilon(\xi, \tau)
\end{aligned}
$$

When using the generalized Glen's law of Equation (19) straightforward integration yields

$$
\text { (o) } \tau=-\sin \gamma(\mathrm{I}-\gamma) \text {, }
$$

(o)

$$
\stackrel{(0)}{\sigma_{y}}=\cos \gamma(\mathbf{I}-Y),
$$

$$
\begin{aligned}
& (\mathrm{o}) \\
& \sigma_{x}
\end{aligned}=\stackrel{(0)}{\sigma_{y}}
$$$$
\stackrel{(0)}{u}=(Y-\mathrm{I})\left\{\frac{2 \sin \gamma}{\mathrm{I}+\mathfrak{a}}\left[\mathrm{a} \eta+n \int_{0}^{\eta} \mathrm{f}(y) \mathrm{d} y\right]+m \phi \sin ^{m} \gamma\right\},
$$$$
\stackrel{(o)}{v}=-\frac{\partial Y}{\partial \xi}\left\{\frac{2 \sin \gamma}{\mathrm{I}+\mathfrak{a}}\left[\frac{\mathfrak{a}}{2} \eta^{2}+n \int_{0}^{\eta} \int_{0}^{\eta^{\prime}} \mathrm{f}(y) \mathrm{d} y \mathrm{~d} \eta^{\prime}\right]+m \phi \sin ^{m} \gamma \eta\right\},
$$

where

$$
\begin{aligned}
\mathrm{f}(y) & =[\sin \gamma(\mathrm{I}-y)]^{n-\mathrm{I}}, \\
\int_{0}^{\eta} \mathrm{f}(y) \mathrm{d} y & =\frac{\mathrm{I}}{n} \sin ^{n-\mathrm{I}} \gamma\left(\mathrm{I}-(\mathrm{I}-\eta)^{n}\right), \\
\int_{0}^{\eta} \int_{0}^{\eta^{\prime}} \mathrm{f}(y) \mathrm{d} y \mathrm{~d} y^{\prime} & =\frac{\sin ^{n-\mathrm{I}} \gamma}{n(n+\mathrm{I})}\left((n+\mathrm{I}) \eta+(\mathrm{I}-\eta)^{n+\mathrm{I}}-\mathrm{I}\right) .
\end{aligned}
$$

There remains the determination of the surface topography. It may be derived from the third of Equations (24) and the resulting Equations (25). One obtains

$$
\frac{\partial \Upsilon}{\partial \tau}+\mathfrak{C}(\Upsilon) \frac{\partial \Upsilon}{\partial \xi}=\frac{\mathfrak{A}}{\mu},
$$

with

$$
\mathfrak{C}=\frac{2 \sin ^{n} \gamma}{\mathrm{I}+\mathfrak{a}}\left[(\mathbf{I}-Y)^{n+\mathbf{I}}+(2 \gamma-\mathrm{I})\right]+\frac{2 \mathfrak{a} \sin \gamma}{\mathrm{I}+\mathfrak{a}} \Upsilon^{2}+m \phi \sin ^{m} \gamma(2 \gamma-\mathbf{I}+\mathbf{I} / m) .
$$

Equation (27) is the desired result. For $\mathfrak{A}=0$ it represents a forward-breaking wave. For any initial disturbance $Y(\xi, 0)=\mathcal{Q}(\xi)$ its solution is

$$
r=\text { 国 }(\xi-\mathbb{C}(Y) \tau) \text {, }
$$


which determines $Y(\xi, \tau)$ implicitly. The resulting Equation (29) implies that a given disturbance will deform with progressing time; the solution must eventually fail as the profile steepens and $\mu$ becomes finite.* The above results do not yet give indications on the unsuitability of Glen's flow law. Indeed we may set $\mathbf{a}=0$ without encountering any formal difficulties. Yet these will arise as soon as terms $O\left(\mu^{2}\right)$ are considered.

The perturbation procedure outlined above yields a sequence of linear non-homogeneous differential equations subject to boundary conditions some of which are non-linear. The zeroth order approximation resulted in the forward-wave equation (29). It can be improved by constructing higher-order approximations. To second order one then obtains

$$
\begin{aligned}
& \frac{\partial \Upsilon}{\partial \tau}+\mathfrak{C} \frac{\partial Y}{\partial \xi}-\mu\left[\mathbb{Z} \frac{\partial^{2} \Upsilon}{\partial \xi^{2}}+\mathfrak{E}\left(\frac{\partial \Upsilon}{\partial \xi}\right)^{2}\right]- \\
& -\mu^{2}\left[\mathfrak{J} \frac{\partial^{2} \Upsilon}{\partial \xi^{3}}+\mathfrak{\xi} \frac{\partial^{2} \Upsilon}{\partial \xi^{2}} \frac{\partial \Upsilon}{\partial \xi}+\mathfrak{H}\left(\frac{\partial Y}{\partial \xi}\right)^{3}\right]+O\left(\mu^{3}\right)=\frac{\mathfrak{A}}{\mu} \text {. }
\end{aligned}
$$

The functions 相, Appendix. The corresponding algebra is unwieldy and becomes unjustifiably involved if terms $O\left(\mu^{3}\right)$ are also considered. $\dagger$ But with the adjusted constitutive relationship for the stress deviator none of the integrals occurring becomes singular, so that all coefficient functions in Equation (30) are bounded.

Analytical solutions to Equation (30) are probably hard to find, yet some insight can be gained for special cases. To this end let us write $Y=\mathrm{I}+\delta \eta$ (since $\eta$ no longer appears we may use this symbol with this new meaning). Then

$$
\begin{aligned}
\frac{\partial \eta}{\partial \tau}+\mathfrak{C}(\mathrm{I} & +\delta \eta) \frac{\partial \eta}{\partial \xi}-\mu\left[\mathbb{Z}(\mathrm{I}+\delta \eta) \frac{\partial^{2} \eta}{\partial \xi^{2}}+\delta \mathfrak{E}(\mathrm{I}+\delta \eta)\left(\frac{\partial \eta}{\partial \xi}\right)^{2}\right]- \\
-\mu^{2} & {\left[\mathfrak{I}(\mathrm{I}+\delta \eta) \frac{\partial^{3} \eta}{\partial \xi^{3}}+\delta \mathfrak{\xi}(\mathrm{I}+\delta \eta) \frac{\partial^{2} \eta}{\partial \xi^{2}} \frac{\partial \eta}{\partial \xi}+\delta^{2} \boldsymbol{I}(\mathbf{I}+\delta \eta)\left(\frac{\partial \eta}{\partial \xi}\right)^{2}\right]=\frac{\mathfrak{A}}{\delta \mu} . }
\end{aligned}
$$

Here, the parameter $\delta$ represents a typical surface elevation above the undisturbed level $Y=\mathrm{I}$. According to the perturbation procedure it cannot be larger than $O(\mu)$ but could be smaller. Furthermore, Equation (3I) gives indications on the size of $\mathfrak{A}$ for which the equation makes sense. Clearly, $\mathfrak{A}$ should not be larger than $O(\mu \delta)$. For real glaciers and ice sheets this condition is well satisfied. In what follows we shall set $\mathscr{A}=\mu \delta \mathfrak{d}$ with $\mathfrak{d}=O(\mathrm{I})$. With such limitations Equation $(3 \mathrm{I})$ may be expressed in powers of $\delta$. To zeroth order the linear longwave theory emerges for which

To first order in $\delta$ we find

$$
\frac{\partial \eta}{\partial \tau}+\mathbb{C}(\mathrm{I}) \frac{\partial \eta}{\partial \xi}-\mu \mathbb{I}(\mathrm{I}) \frac{\partial^{2} \eta}{\partial \xi^{2}}-\mu^{2} \mathfrak{I}(\mathrm{I}) \frac{\partial^{3} \eta}{\partial \xi^{3}}=\mathfrak{U} .
$$

$$
\begin{aligned}
\frac{\partial \eta}{\partial \tau}+\mathbb{C}(\mathrm{I}) \frac{\partial \eta}{\partial \xi}-\mu \mathbb{I}(\mathrm{I}) & \frac{\partial^{2} \eta}{\partial \xi^{2}}-\mu^{2} \mathfrak{I}(\mathrm{I}) \frac{\partial^{3} \eta}{\partial \xi^{3}}+ \\
+\delta\left\{\mathbb{C}^{\prime}(\mathrm{I}) \eta \frac{\partial \eta}{\partial \xi}-\mu\right. & {\left[\mathbb{Z}^{\prime}(\mathrm{I}) \eta \frac{\partial^{2} \eta}{\partial \xi^{2}}+\mathbb{E}(\mathrm{I})\left(\frac{\partial \eta}{\partial \xi}\right)^{2}\right]-} \\
& \left.-\mu^{2}\left[\mathfrak{J}^{\prime}(\mathrm{I}) \eta \frac{\partial^{3} \eta}{\partial \xi^{3}}+\mathfrak{G}(\mathrm{I}) \frac{\partial^{2} \eta}{\partial \xi^{2}} \frac{\partial \eta}{\partial \xi}\right]\right\}=\mathfrak{H} .
\end{aligned}
$$

* That this must be so for $\mathbb{C}$ 's which are monotonically increasing with $Y$ (and this can be shown to be the case here, see Appendix) can be seen as follows: $\mathfrak{C}$ is a disturbance velocity which grows with growing $Y$. A disturbance of sinusoidal shape will therefore steepen as wave heights travel faster than points of zero elevation. Small wavelengths of a Fourier transform of this new wave form will grow in significance changing thereby the magnitude of $\mu$ which eventually will become finite.

$\dagger$ Since equations have been linearized with regard to stresses, we do not believe that terms $O\left(\mu^{3}\right)$ would give any physical improvement. 
Nothing can be inferred about the size of the various coefficients involved. In particular they need not be $O(\mathrm{r})$, because they follow from the integrations performed in the Appendix. The remaining simplifications therefore depend on the order of magnitude of the various terms considered.

(i) If $\mathbb{Z}(\mathrm{I})=O(\mathrm{I})$ and $\delta=O(\mu)$ the leading terms are

$$
\frac{\partial \eta}{\partial \tau}+\mathbb{C}(\mathrm{I}) \frac{\partial \eta}{\partial \xi}-\mu \text { 迅 (I) } \frac{\partial^{2} \eta}{\partial \xi^{2}}+\delta \mathbb{C}^{\prime}(\mathrm{I}) \eta \frac{\partial \eta}{\partial \xi}=\mathbb{d} \text {. }
$$

(ii) If 咽(I) $=O(\mathrm{I})$ and $\delta=O\left(\mu^{2}\right)$ then

$$
\frac{\partial \eta}{\partial \tau}+\mathbb{C}(\mathrm{I}) \frac{\partial \eta}{\partial \xi}-\mu \mathbb{\mathbb { A }}(\mathrm{I}) \frac{\partial^{2} \eta}{\partial \xi^{2}}-\mu^{2} \mathfrak{J}(\mathrm{I}) \frac{\partial^{3} \eta}{\partial \xi^{3}}+\delta \mathbb{C}^{\prime}(\mathrm{I}) \eta \frac{\partial \eta}{\partial \xi}=\mathfrak{d} \text {. }
$$

(iii) If $\mathbb{Z}$ (I) $\mathrm{I})=O\left(\mu^{-1}\right)$, 歶 $(\mathrm{I})=O\left(\mu^{-1}\right)$ the leading terms are

$$
\frac{\partial \eta}{\partial \tau}+\mathbb{C}(\mathrm{I}) \frac{\partial \eta}{\partial \xi}-\mu \mathbb{\mathbb { A }}(\mathrm{I}) \frac{\partial^{2} \eta}{\partial \xi^{2}}+\delta\left[\mathbb{C}^{\prime}(\mathrm{I}) \eta \frac{\partial \eta}{\partial \xi}-\mu \mathbb{\mathbb { I }}^{\prime}(\mathrm{I}) \eta \frac{\partial^{2} \eta}{\partial \xi^{2}}\right]=\mathfrak{d} .
$$

Clarity about which of these equations should apply is obtained if the magnitude of the various coefficient functions is determined. First indications follow, if we assume no slip, $\phi=0$, and use Glen's flow law, $\mathfrak{a}=0$, for which $\mathbb{C}$ and $\mathbb{Z}$ are still well defined. The Appendix then shows that

$$
\frac{\mathbb{C}(\mathrm{I})}{\sin \gamma}=2, \quad \frac{\mathbb{C}^{\prime}(\mathrm{I})}{\mathbb{C}(\mathrm{I})}=2, \quad \frac{\mathbb{I}(\mathrm{I})}{\mathbb{C}(\mathrm{I})}=\frac{d}{2} \cot \gamma,
$$

with $d=\left(\frac{1}{3}, \frac{1}{2}, \frac{3}{5}\right)$ for $n=(1,2,3)$. Thus, as long as $\gamma=O(\mathrm{I})$ and as long as wavelengths are sufficiently large the simple forward-wave equation $(27)$ is a reasonable approximation. It can be improved by allowing moderately long wavelengths. Dependent on the magnitude of surface elevation, Equations (32), (34), or (35) apply. For small inclinations, on the other hand, cot $\gamma$ becomes large and diffusion is no longer negligible. The perturbation procedure still remains valid in this case, because the steady-state solution (8) does not depend on it. In contrast to steep valley glaciers, diffusion is therefore never negligible in ice sheets, because in these $\gamma \ll \mathrm{I}$. A model equation for these is either Equation (32) of the linear long-wave theory or Equations (34) and $(36)$ if first-order corrections to surface elevations are taken into account.

\section{Solutions of the governing equations}

When compared with the kinematic wave theory as presented by Nye, none of the above equations matches fully with his own which is

$$
\frac{\partial \eta}{\partial \tau}+\left(C_{0}-D_{0}{ }^{\prime}\right) \frac{\partial \eta}{\partial \xi}-D_{0} \frac{\partial^{2} \eta}{\partial \xi^{2}}+C_{0}{ }^{\prime} \eta=\boldsymbol{q} \text {. }
$$

Here, $C_{0}, C_{0}{ }^{\prime}=\mathrm{d} C_{0} / \mathrm{d} \xi, D_{0}$, and $D_{0}{ }^{\prime}=\mathrm{d} D_{0} / \mathrm{d} \xi$ are known functions of $\xi$. Differences between Equations (32) and (37) evolve because Equation (37) is valid for a slope of ice with variable thickness, whereas Equation (32) was derived for a slab of constant thickness, and, clearly, because Equation $\left(3^{2}\right)$ contains $O\left(\mu^{2}\right)$ terms. For steep slopes their neglect is justified. Solutions for this case are of limited interest, but follow most easily if Equation (32) is subject to the Galilean transformation

$$
X=\frac{\xi-\mathbb{C}(\mathrm{I}) \tau}{(\mu \mathbb{Z}(\mathrm{I}))^{\frac{1}{2}}}, \quad T=\tau .
$$


We then obtain the heat equation,

$$
\frac{\partial \eta}{\partial T}-\frac{\partial^{2} \eta}{\partial X^{2}}=\mathfrak{d}(X-\mathfrak{C}(\mathrm{I}) T, T)=\hat{\mathfrak{U}}(X, T),
$$

with the general solution

$$
\begin{aligned}
\eta(X, T) & =\int_{T_{0}=0}^{T_{0}=T} \mathrm{~d} T_{0} \int_{-\infty}^{\infty} \frac{\hat{\hat{H}}\left(X_{0}, T_{0}\right)}{\left[4 \pi\left(T-T_{0}\right)\right]^{\frac{1}{2}}} \\
& \exp \left\{-\frac{\left(X-X_{0}\right)^{2}}{4\left(T-T_{0}\right)}\right\} \mathrm{d} X_{0}+ \\
& +\int_{-\infty}^{\infty} \frac{\eta_{0}\left\{(\mu \mathbb{T}(\mathrm{I}))^{\frac{1}{2}} X_{0}\right\}}{(4 \pi T)^{\frac{1}{2}}} \exp \left\{-\frac{\left(X-X_{0}\right)^{2}}{4 T}\right\} \mathrm{d} X_{0},
\end{aligned}
$$

in which $\eta_{0}(\xi)$ is the initial disturbance.

When applying Equation (38) to the non-linear Equation (34) what obtains is the inhomogeneous Burger equation (see, e.g. Leibowich and Seebass, 1974)

$$
\frac{\partial \eta}{\partial \tau}-\frac{\partial^{2} \eta}{\partial X^{2}}+\kappa \eta \frac{\partial \eta}{\partial X}=\hat{\imath}(X, T)
$$

where

$$
\kappa=\frac{\delta}{\mu^{\frac{1}{2}}} \frac{\mathbb{C}^{\prime}(\mathrm{I})}{\mathbb{J}(\mathrm{I})^{\frac{1}{2}}} .
$$

Construction of solutions to Equation (4I) depends on the magnitude of $\kappa$. If $\kappa$ is large, small perturbation solutions are suggested. If $\kappa=O(\mathrm{I})$ and, more generally, for all $\kappa$, solutions are determined by utilizing the Hopf-Cole transformation

$$
\psi=\exp \left\{\frac{\kappa}{2} \int_{X}^{\infty} \eta\left(X^{\prime}, T\right) \mathrm{d} X^{\prime}\right\} \rightarrow \eta=-\frac{2}{\kappa} \frac{\partial \psi / \partial X}{\psi},
$$

which reduces Equation $(4 \mathrm{r})$ to the linear equation

$$
\frac{\partial \psi}{\partial T}-\frac{\partial^{2} \psi}{\partial X^{2}}+\left(\frac{\kappa}{2} \int_{0}^{X} \hat{\alpha}\left(X^{\prime}, T\right) \mathrm{d} X^{\prime}\right) \psi=0 .
$$

For arbitrary accumulation-rate it must be integrated numerically. For impulsive accumulation-rate

$$
\hat{\mathrm{A}}=\hat{a}(X-T) \delta(T),
$$

it can, however, be treated exactly, because then Equation (43) reduces to the heat equation. Thus we can solve the initial-value problem to find

$$
\eta(X, T)=\frac{\mathrm{I}}{\kappa} \frac{\int_{-\infty}^{\infty} \frac{X-X^{\prime}}{T} \exp \left[\frac{\kappa}{2} \int_{X^{\prime}}^{\infty} \hat{a}\left(X^{\prime \prime}\right) \mathrm{d} X^{\prime \prime}-\frac{\left(X-X^{\prime}\right)^{2}}{4 T}\right] \mathrm{d} X^{\prime}}{\int_{-\infty}^{\infty} \exp \left[\frac{\kappa}{2} \int_{X^{\prime}}^{\infty} \hat{a}\left(X^{\prime \prime}\right) \mathrm{d} X^{\prime \prime}-\frac{\left(X-X^{\prime \prime}\right)^{2}}{4 T}\right] \mathrm{d} X^{\prime \prime}},
$$

which provides the detailed structure of the wave for all time; $\hat{a}(X)$ is identical with the initial disturbance of the surface elevation above its mean. 
If $\kappa$ is small, multiple time scales are suggested, but these are not needed, because when solutions to Equation (4I) are constructed by the regular perturbation expansion

$$
\eta=\sum_{j=0}^{\infty} \kappa^{j} \eta,
$$

no secular terms arise. What obtains is a sequence of heat equations

$$
\frac{\partial \eta}{\partial T}-\frac{\partial^{2} \eta}{\partial X^{2}}=\left\{\begin{array}{cc}
\hat{\mathrm{H}}(X, T), & j=\mathrm{o}, \\
-\sum_{\mu=0}^{j-\mathrm{I}} \eta \frac{(\mu)}{\eta(j-\mu)} \frac{\partial \eta}{\partial X}, & j \geqslant \mathrm{I},
\end{array}\right.
$$

of which solutions again have the form of Equation (40) and will not be repeated here. If $O\left(\mu^{2}\right)$ terms are included in an exploitation of Equation (32) the solution may again be written in convolution form

$$
\begin{aligned}
\eta(X, T)=\int_{T_{0}=0}^{T_{0}=T} \mathrm{~d} T_{0} \int_{-\infty}^{\infty} \mathscr{G}\left(X-X_{0}, T-T_{0}\right) \hat{\mathrm{A}}\left(X_{0}, T_{0}\right) \mathrm{d} X_{0}+ & \\
& +\int_{-\infty}^{\infty} \mathscr{G}\left(X-X_{0}, T\right) \eta_{0}\left(X_{0}\right) \mathrm{d} X_{0}
\end{aligned}
$$

where the Green's function $\mathscr{G}$ is given by

$$
\begin{aligned}
\mathscr{G}(X, T) & =\frac{\mathrm{I}}{2 \pi} \int_{-\infty}^{\infty} \exp \left\{\mathrm{i} s X-\left[s^{2}-\left(\mu^{\frac{1}{2}} \mathfrak{J}(\mathrm{I}) / \mathbb{T}(\mathrm{I})^{\frac{3}{2}}\right) \text { is }\right] T\right\} \mathrm{d} s \\
& =\frac{\mathrm{I}}{(4 \pi T)^{\frac{1}{2}}} \exp \left\{-\frac{\left(X+\left(\mu^{\frac{1}{2}} \mathfrak{J}(\mathrm{I}) / \mathbb{T}(\mathrm{I})^{\frac{3}{2}}\right) T\right)^{2}}{4 T}\right\},
\end{aligned}
$$

which for $\mu=0$ reduces to Equation (40). When

$$
\mathrm{I} / \epsilon=\mu^{\frac{1}{2}} \mathfrak{J}(\mathrm{I}) / \mathbb{Z}\left(\mathrm{I}(\mathrm{I})^{\frac{3}{2}} \ll \mathrm{I},\right.
$$

a perturbation solution is suggested. If boundaries are at infinity this yields a hierarchy of heat equations, namely, if

$$
\begin{aligned}
\eta & =\sum_{\nu=0}^{\infty}\left(\frac{\mathrm{I}}{\epsilon}\right)^{\nu(\nu)}, \\
\frac{\partial \eta}{\partial T}-\frac{\partial^{2} \eta}{\partial X^{2}} & = \begin{cases}\hat{\mathrm{A}}(X, T), & j=0, \\
\partial^{(j} \eta & \\
\frac{\partial^{(j)}}{\partial X^{3}}, & j \geqslant \mathrm{I},\end{cases}
\end{aligned}
$$

of which the solutions have again the form of Equation (40).

For any other more complicated equation, say Equations (35) or (36) direct numerical methods must be suggested for their solution.

\section{Discussion AND Gonclusion}

The calculations performed above remain rather theoretical and are of little help for real glaciological problems as long as no numerical values for the various coefficients of the surface-wave equation are available. Since our model treats surface waves on a plane parallelsided slab, it will be of value for a glacier in the regions far from its head or its snout. 
Several effects are built into the model:

(i) finite inclination of the ice slab,

(ii) sliding of the ice over its bed,

(iii) generalization of Glen's flow law.

It is interesting to see how these effects influence the coefficients in the basic governing equations. All these can be summarized in the equation

or

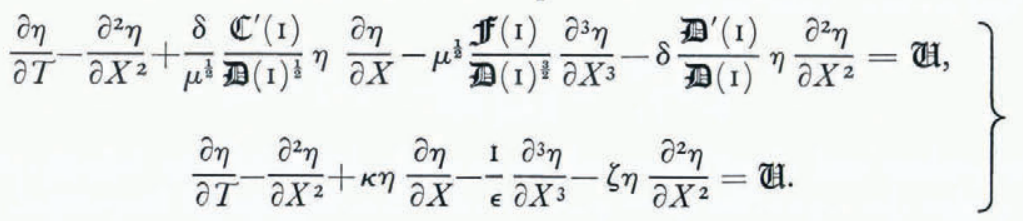

The first two terms on the left-hand side represent essentially the kinematic wave theory including diffusion. In the original coordinates $\xi$ and $\tau$ the corresponding equation contains two constants, $\mathbb{C}(\mathrm{I})$ and $\mathbb{Z}(\mathrm{I})$, which are now incorporated in the variable $X$. In Figures $\mathrm{I}$ and 2 these two coefficients are plotted against the inclination angle $\gamma$. Curves are shown for two values, namely $n=2$ and $n=3$, of the exponent in Glen's flow law; they are, furthermore, parameterized for different values of the coefficient a in the generalized Glen law introduced in Equation (19). Figures ra and 2a hold for an ice slope adhering to its bed; in Figures $\mathrm{Ib}$ and $2 \mathrm{~b}$ two sliding velocities have been introduced, namely $u_{\mathrm{b}}=0.005$ and $u_{\mathrm{b}}=$ o.or. (Notice that this corresponds to a sliding parameter $\phi=u_{\mathrm{b}} / \sin ^{m} \gamma$; hence, keeping $u_{\mathrm{b}}$ fixed means that $\phi$ changes with $\gamma$. Calculations were performed for $m=2$.) It is seen that the generalization of Glen's flow law has little effect on the numerical values of

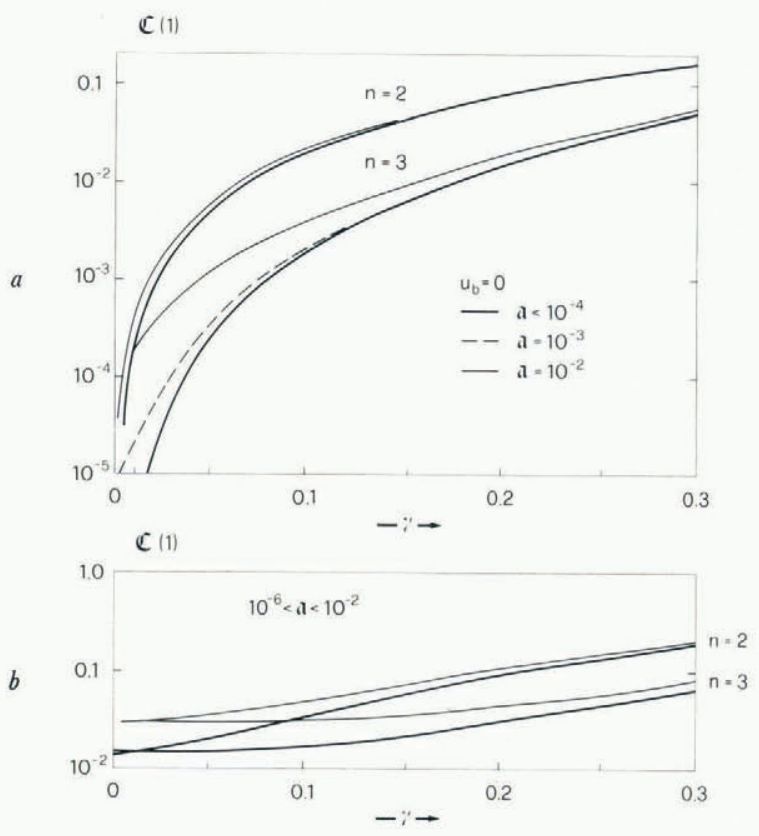

Fig. I.

(a) Surface-wave velocity $\mathbb{C}(I)$ as a function of the inclination angle $\gamma$ parameterized for $n=2,3$ and various values of a in the generalized Glen flow law. No slip of the glacier at the bed.

(b) Same as Figure Ia, but for the case when sliding occurs. The sliding law is $u_{\mathrm{b}}=\phi \tau^{m}$, and has been used for $m=2$. The thick lines are for $u_{\mathrm{b}}=0.005$, the thin lines for $u_{\mathrm{b}}=0.01$. 
$\mathbb{C}$ and $\mathbb{Z}$. This is no surprise, because the expressions for $\mathbb{C}$ and $\mathbb{Z}$ show no singularity when $\mathfrak{a}=0$. The dependence on the inclination angle $\gamma$, the exponent $n$, and the sliding velocity are more important. To find the range of importance of diffusion we write Equation (32) with respect to the variables

$$
\bar{\xi}=\xi / \mathbb{C}(\mathrm{I}), \tau
$$

and then find to first order in $\mu$

$$
\frac{\partial \eta}{\partial \tau}+\frac{\partial \eta}{\partial \xi}-\mu \frac{\mathbb{Z}(\mathrm{I})}{\mathfrak{C}(\mathrm{I})} \frac{\partial^{2} \eta}{\partial \xi^{2}}+O\left(\mu^{2}\right)=\mathfrak{d} .
$$

For Glen's flow law and when the ice adheres to the bed it was shown that $\mathbb{D}(\mathrm{I}) / \mathbb{C}(\mathrm{I}) \propto \cot \gamma$. For small angles (in reality for most glaciers) diffusion is therefore important. Whether this persists also when sliding over the bed is allowed for, can easily be inferred from Figures $\mathrm{Ib}$ and 2 b. Accordingly, $\mathbb{\mathbb { B }}(\mathrm{I}) / \mathbb{C}(\mathrm{I})$ grows monotonically with decreasing $\gamma$. Numerical calculations indicate that with sufficient accuracy one may set $\mathbb{Z}(\mathrm{I}) / \mathbb{C}(\mathrm{I})=0.6 \cot \gamma$. Hence, even when sliding occurs, diffusion remains significant at all inclination angles which occur.

Diffusion being significant we shall henceforth and irrespective of what value the wavelength parameter may have, use $X$ and $T$ as independent variables. With their use a possible extension of kinematic wave theory follows from numerical values of $\mathbb{C}^{\prime}(\mathrm{I}) / \mathbf{I}(\mathrm{I})^{\frac{1}{2}}$,

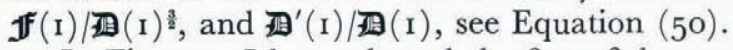

In Figure $3 \mathrm{I}$ have plotted the first of these parameters as a function of $\gamma$, parameterized for different values of $\mathbf{a}$. In Figure 3 a the no-slip condition has been applied, whereas Figure $3^{\mathrm{b}}$ shows the corresponding results including sliding. Generally, sliding enhances the value of $\mathbb{C}^{\prime}(\mathrm{I}) / \mathbb{T}(\mathrm{I})^{\frac{1}{2}}$ at small inclinations, but has practically no influence on it at larger inclinations
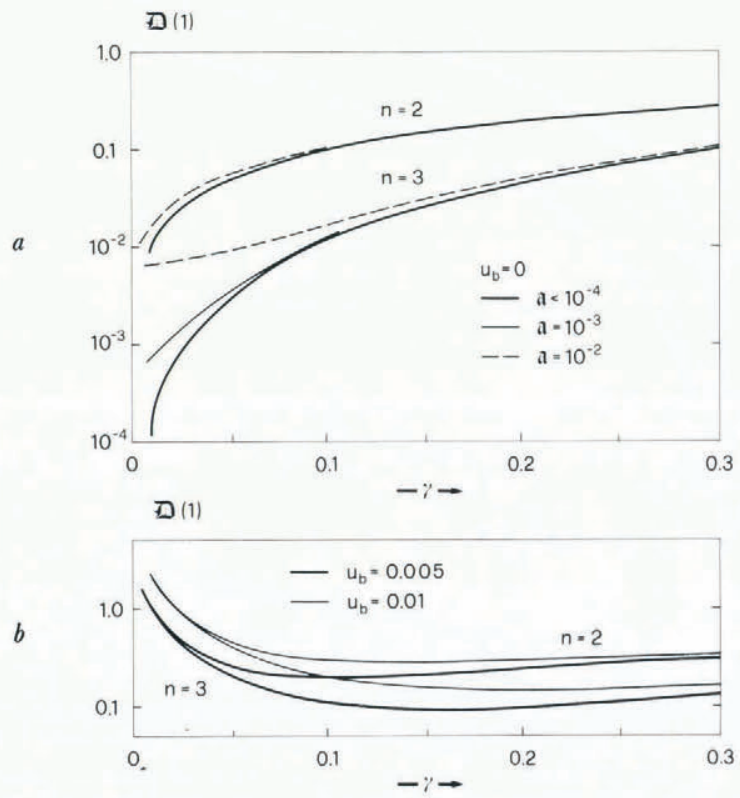

Fig. 2.

(a) Diffusion parameter $\mathbb{I}(I)$ as a function of the inclination angle $\gamma$ parameterized for $n=2,3$ and for various values of a in the generalized Glen flow law. No slip of the glacier at the bed.

(b) Same as Figure 2a, but for the case when sliding occurs. The sliding law is $u_{\mathrm{b}}=\phi \tau^{m}, m=2$. 
$\gamma$. In view of the definition of $\kappa$ and since for most inclinations $\gamma$ occurring in practice $\left|\mathbb{C}^{\prime}(\mathrm{I}) / \mathbb{I}(\mathrm{I})^{\frac{1}{2}}\right|=O\left(\mathrm{IO}^{-1}\right)$ I conclude that the non-linear term $\eta \partial \eta / \partial X$ in Equation (50) need not be taken into account except, perhaps, in steep and hanging glaciers for which $\kappa$ may be of the same order as usual diffusion parameters. This result can be strengthened if the steady-state Burgers' equation is looked at and the corresponding shock thickness is evaluated, see Lick (I970). One finds that the latter is

$$
\xi_{0}=\frac{2}{\kappa} \frac{\mathrm{I}}{\eta_{0}-\mathrm{I}},
$$

where $\eta_{0}$ is the thickness behind the shock. Generally it must be expected to be close to unity so that $\eta_{0}-\mathrm{I} \ll \mathrm{I}$. Hence, even with $\kappa=O(\mathrm{I}), \xi_{0}$ is large and under usual circumstances greater than the length of the glacier. The non-linear term associated with $\kappa$ is therefore negligible.

The significance of the coefficient $\epsilon$ in Equation (50) can be estimated from Figure 4 . (Effects due to this term are usually attributed to dispersion.) Unlike the functions of the previous graphs, the value of $\mathbb{f}(\mathrm{I}) / \mathbb{R}(\mathrm{I})^{\frac{3}{2}}$ strongly depends on the parameter $\mathfrak{a}$ in the generalized Glen law. The reason, clearly, is that $\mathfrak{f}(\mathbf{I}) \rightarrow \infty$ as $\mathfrak{a} \rightarrow 0$. For a glacier adhering to its bed $\mathfrak{I}(\mathrm{I}) / \mathrm{I}(\mathrm{I})^{\frac{3}{2}}$ grows with growing $\gamma$ and is, except for very small angles $\gamma$, of the order of $\mathrm{IO}^{3}-\mathrm{IO}^{0}$. When sliding takes place $\mathfrak{1}(\mathrm{I}) / \mathrm{I}(\mathrm{I})^{\frac{3}{2}}$ increases also as $\gamma \rightarrow 0$. Here we may uniformly say that $\mathfrak{f}(\mathrm{I}) / \boldsymbol{I}(\mathrm{I})^{\frac{3}{2}}$ is large. Its value depends also rather critically on the value of the coefficient $n$. We conclude that under no circumstances is it allowable to neglect in Equation (50) the term involving $\epsilon$, because under usual situations $\epsilon$ is of the order of $10^{0}$ and larger. Furthermore, $\epsilon$ may exceed the value $10^{\circ}$ by several orders of magnitude. Consequently, dispersion may very well be of greater significance in glaciers than is diffusion.

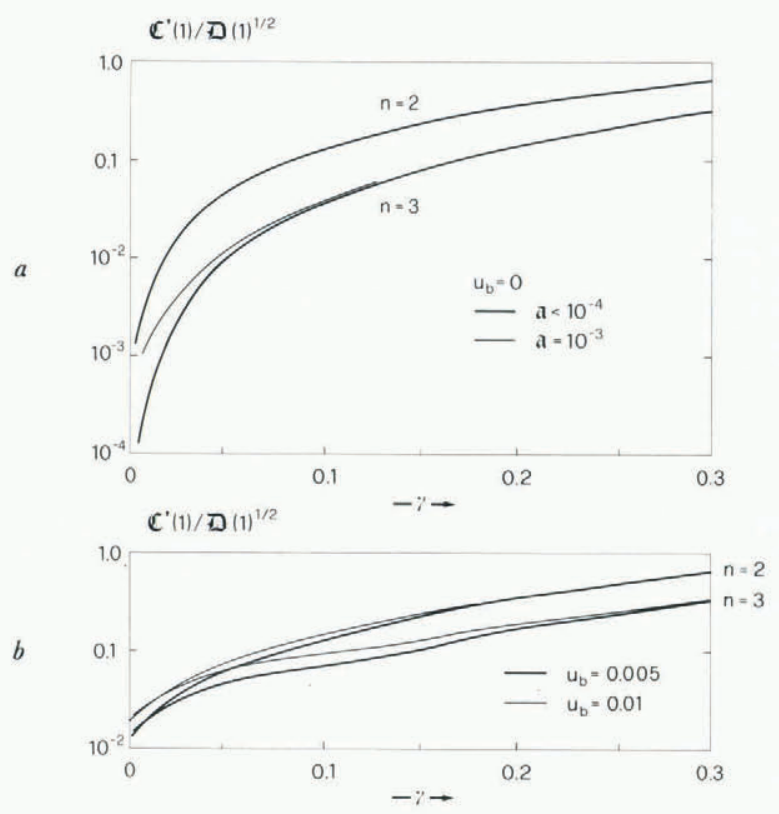

Fig. 3 .

(a) $\mathbb{Z}^{\prime}(I) / \mathbb{C}(I)^{1}$ as a function of the inclination angle $\gamma$ parameterized for $n=2,3$ and various values of a in the generalized Glen flow law. No slip of the glacier at the bed.

(b) Same as Figure $3 a$, but for the case when sliding occurs. The sliding law is $u_{\mathrm{b}}=\phi \tau^{m}, m=2$. 


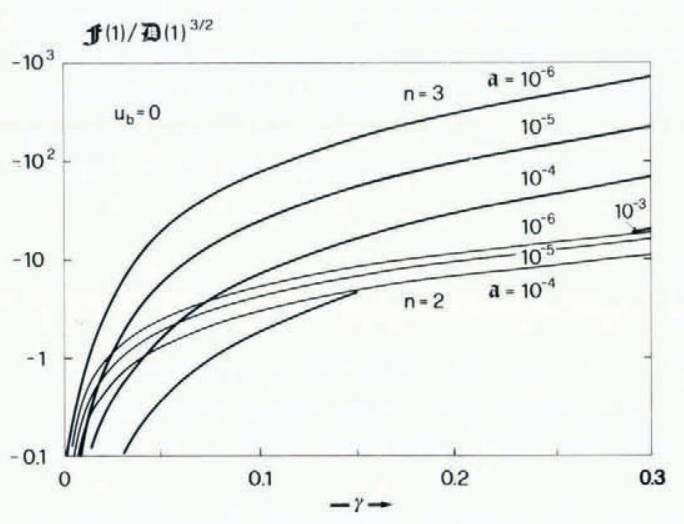

$a$

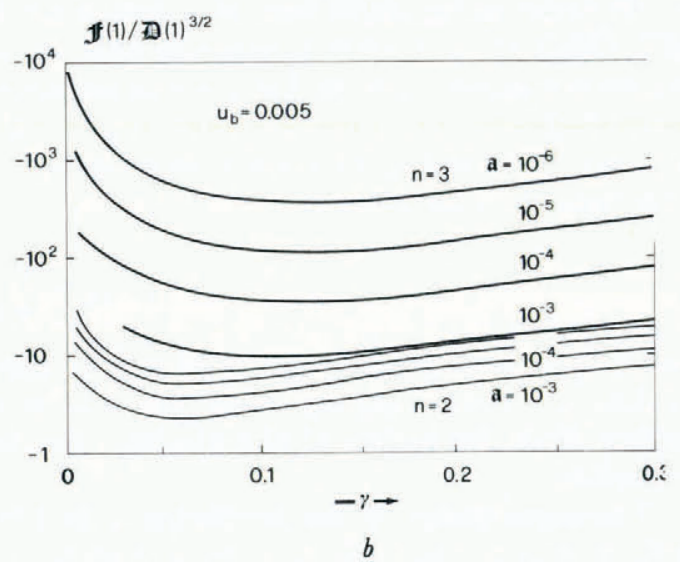

b

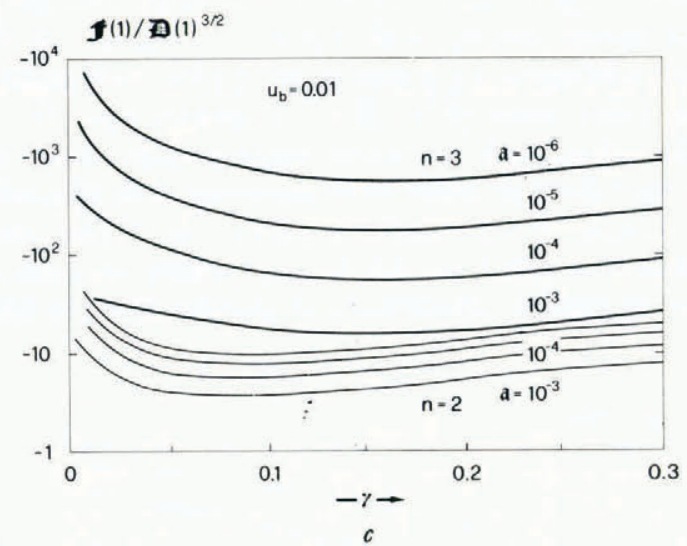

Fig. 4 .

(a) Dispersion parameter $\mathfrak{I}(I) / \mathbb{A}(I)^{3 / 2}$ plotted against the inclination angle $\gamma$ parameterized for $n=2,3$ and various values of a. No slip of the glacier at the bed.

(b) Same as Figure $4 a$, but for a sliding velocity $u_{\mathrm{b}}=0.005$. The sliding law is $u_{\mathrm{b}}=\phi \tau^{m}, m=2$.

(c) Same as Figure $4 a$, but for a sliding velocity $u_{\mathrm{b}}=0.01$. The sliding law is $u_{\mathrm{b}}=\phi \tau^{m}, m=2$.

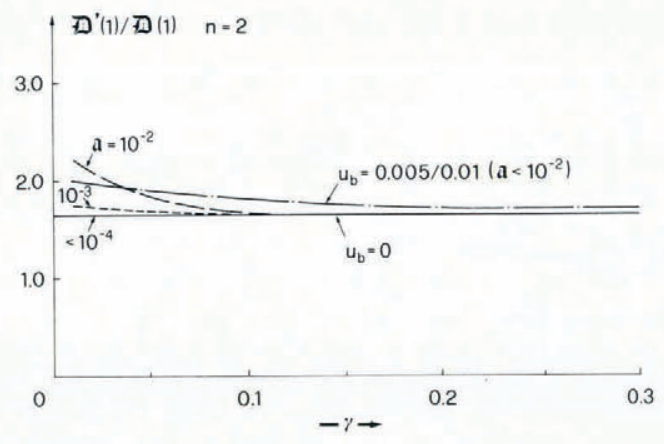

$a$

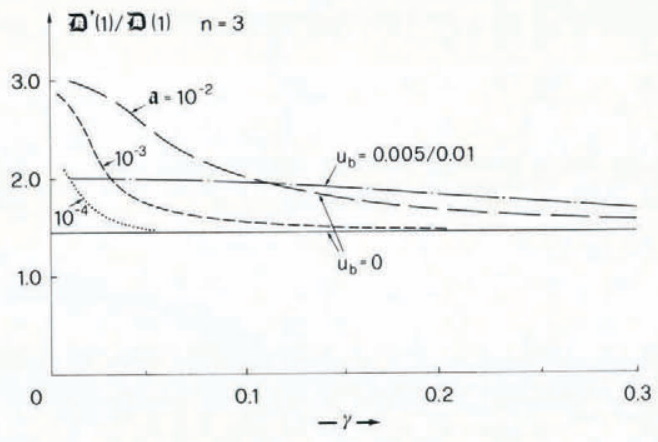

$b$

Fig. 5 .

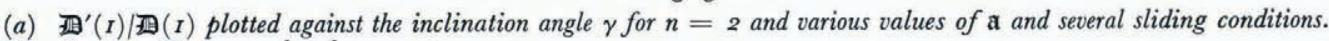

(b) Same as Figure 5a, but for $n=3$. 
Finally, in order to obtain information about the magnitude of $\mathbb{Z}^{\prime}(\mathrm{I}) / \mathbb{\text { I }}(\mathrm{I})$, I have plotted in Figure 5 this ratio as a function of $\gamma$ and parameterized for various values of $n$ and a. It is seen that, uniformly, $\mathrm{IO}^{\circ}<\mathbb{Z}^{\prime}(\mathrm{I}) / \mathbb{\mathbb { }}(\mathrm{I})<\mathrm{IO}^{\mathrm{I}}$ so that $\zeta$ is small of order $1 \mathrm{IO}^{-2}$ or smaller.

In conclusion we may say that the surface-wave equation appropriate for ice slopes must have the form

$$
\frac{\partial \eta}{\partial T}-\frac{\partial^{2} \eta}{\partial X^{2}}+\frac{\mathrm{I}}{\epsilon} \frac{\partial^{3} \eta}{\partial X^{3}}+\kappa \eta \frac{\partial \eta}{\partial X}-\zeta \eta \frac{\partial^{2} \eta}{\partial X^{2}}=\boldsymbol{d},
$$

where for ice $I / \epsilon$ is the dominant coefficient and where $|\zeta|$ is generally larger than $|\kappa|$, but smaller than unity. The non-linear terms in Equation $\left(5^{\circ}\right)$ can safely be regarded as small corrections of the linear reduced equation, in which dispersion and diffusion are taken into account. The non-linear terms can safely be neglected so that the zeroth order equation reads

$$
\frac{\partial \eta}{\partial T}-\frac{\partial^{2} \eta}{\partial X^{2}}+\frac{1}{\epsilon} \frac{\partial^{3} \eta}{\partial X^{3}}=\mathfrak{d} \text {. }
$$

Of the diffusion and dispersion effects contained in this equation the second is more important than the former but this is a materially dependent property for which dependence on the parameter $\mathbf{a}$ is evidenced (see Fig. 4). For materials with a sufficiently pronounced linear behaviour of the stress-strain-rate relationship (that is for $n=3$ and $\mathfrak{a}>10^{-1}$ ), $\epsilon$ becomes large; in this instance diffusion overrides dispersion and may perhaps, even be negligible. For ice this does not appear to be the case, since for $n=3, \mathrm{a}<c$. $\mathrm{Io}^{-3}$. Consequently, kinematic wave theory is inappropriate for modelling surface-wave phenomena on ice.

MS. received 12 December 1978 and in revised form 17 May 1979

\section{APPENDIX}

Here we briefly summarize the derivation of Equation (30). To this end the Equations (12)-(14) are written for the $O(\mu)$ terms. The differential equations for the stresses are

$$
\frac{\partial \tau}{\partial \eta}=-\frac{\partial \sigma_{x}}{\partial \xi}=\cos \gamma \frac{\partial r}{\partial \xi}, \quad \frac{\partial \sigma_{y}}{\partial \eta}=-\frac{\partial \tau}{\partial \xi}=-\sin \gamma \frac{\partial r}{\partial \xi},
$$

and must satisfy the boundary conditions (see Equation (14))

$$
\stackrel{(\mathrm{r})}{\tau}=\mathrm{o}, \quad \stackrel{(\mathrm{r})}{\sigma_{y}}=\mathrm{o},
$$

and $y=\Upsilon(x, t)$. Straightforward integration thus reveals

$$
\stackrel{(\mathrm{r})}{\tau}=\cos \gamma \frac{\partial Y}{\partial \xi}(\eta-Y), \quad \stackrel{(\mathrm{I})}{\sigma_{y}}=\sin \gamma \frac{\partial \Upsilon}{\partial \xi}(Y-\eta) .
$$

(1)

The longitudinal stresses $\sigma_{x}$ follow from the fourth of Equations (12) and Equation (A.3)

so that

$$
\left.\frac{\partial u}{\partial \xi}=\frac{1}{2}\right]\left(\sin ^{2} \gamma(\mathrm{I}-\eta)^{2}\right)\left(\begin{array}{l}
(\mathrm{r}) \\
\left(\sigma_{x}-\sigma_{y}\right)
\end{array}\right.
$$

$$
\stackrel{(\mathrm{r})}{\sigma_{x}}=\frac{\partial \Upsilon}{\partial \xi}\left\{\sin \gamma(\Upsilon-\eta)+\frac{4 \sin \gamma}{\mathrm{f}(\eta)+\mathbf{a}}\left[\mathbf{a} \eta+\sin ^{n-1} \gamma\left(\mathrm{r}-(\mathrm{r}-\eta)^{n}\right)\right]+m \phi \frac{2(\mathrm{r}+\mathbf{a})}{\mathrm{f}(\eta)+\mathbf{a}} \sin ^{m} \gamma\right\} .
$$

It is now evident that a non-vanishing coefficient a prevents the first-order longitudinal stresses becoming infinite at $\eta=1$. This demonstrates that the generalization of Glen's flow law is important.

To obtain the first-order velocity field the fifth and third of Equations (12) must be integrated. From the former and the first of Equations (A.3) we obtain

$$
\frac{\partial u}{\partial \eta}=\frac{2}{\mathrm{I}+\mathbf{a}}\left\{\mathbf{a}+n[\sin \gamma(\mathrm{I}-\eta)]^{n-1}\right\} \cos \gamma \frac{\partial \Upsilon}{\partial \xi}(\eta-Y) \text {. }
$$


Integrating this and using the boundary condition

gives as first-order longitudinal velocities

$$
\stackrel{\text { (1) }}{u_{\eta=0}}=-\phi m \sin ^{m-1} \gamma \cos \gamma \Gamma \frac{\partial r}{\partial \xi},
$$

$$
\stackrel{(\mathrm{r})}{u}=\stackrel{(\mathrm{r})}{=}{ }_{\mathrm{II}}(\eta, r) \frac{\partial Y}{\partial \xi},
$$

with

$$
\begin{aligned}
\stackrel{(\mathrm{I})}{U}_{\mathrm{II}}(\eta, r)=\cos \gamma\left\{\frac { 2 } { \mathrm { I } + \mathfrak { a } } \left[\mathbf{a}\left(\frac{\eta^{2}}{2}-\eta \gamma\right)+\sin ^{n-1} \gamma(\mathrm{I}-\eta)^{n}\right.\right. & \left(\gamma-\eta-\frac{\mathrm{I}-\eta}{n+\mathrm{I}}\right)+ \\
& \left.\left.+\sin ^{n-1} \gamma\left(\frac{\mathrm{I}}{n+\mathrm{I}}-\gamma\right)\right]-\phi m \sin ^{m-1} \gamma r\right\} .
\end{aligned}
$$

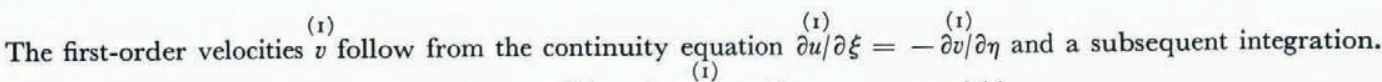
Using Equation (A.6) and the boundary condition that $v$ vanishes at $\eta=0$ yields

$$
\stackrel{(1)}{v=}-\stackrel{(1)}{V}_{11}(\eta, r) \frac{\partial^{2} r}{\partial \xi^{2}}+\stackrel{(1)}{V_{12}}(\eta, r)\left(\frac{\partial r}{\partial \xi}\right)^{2},
$$

where

$$
\begin{aligned}
& \stackrel{(\mathrm{I})}{V}_{\mathrm{II}}(\eta, Y)=\cos \gamma\left\{\frac{2}{\mathrm{I}+\mathrm{a}}[\mathrm{a}\right.\left(\frac{\eta^{3}}{6}-\frac{\eta^{2} \eta}{2}\right)+\frac{\sin ^{n-1} \gamma}{n+\mathrm{I}}\left\langle\left(\gamma-\frac{\mathrm{I}}{n+\mathrm{I}}\right)\left(\mathrm{I}-(\mathrm{I}-\eta)^{n+1}\right)-\right. \\
&\left.-\left(\mathrm{I}-\frac{\mathrm{I}}{n+\mathrm{I}}\right)\left(\frac{\mathrm{I}-(\mathrm{I}-\eta)^{n+2}}{n+2}-\eta(\mathrm{I}-\eta)^{n+\mathrm{I}}\right)\right\rangle+ \\
&\left.\left.\quad+\sin ^{n-1} \gamma\left(\frac{\mathrm{I}}{n+\mathrm{I}}-\gamma\right) \eta\right]-\phi m \sin ^{m-1} \gamma \Upsilon_{\eta}\right\},
\end{aligned}
$$

and

$$
\stackrel{(1)}{V}_{12}(\eta, r)=\cos \gamma\left\{\frac{2}{\mathrm{I}+\mathfrak{a}}\left[\mathfrak{a} \frac{\eta^{2}}{2}-\frac{\sin ^{n-1} \gamma}{n+\mathrm{I}}\left(\mathrm{I}-(\mathrm{I}-\eta)^{n+\mathrm{I}}\right)+\sin ^{n-1} \gamma \eta\right]+\phi m \sin ^{m-1} \gamma \eta\right\} .
$$

This completes the first-order solution; the results determine the wave equation (20) as far as $O(\mu)$ terms go. To determine also the $O\left(\mu^{2}\right)$ terms, second-order shear stresses and velocities must be determined. The former follow from the first of Equations (12), or

$$
\begin{aligned}
\frac{\partial \tau}{\partial \eta}=-\frac{\partial \sigma_{x}}{\partial \xi}=-\sin \gamma\left(\frac{\partial \Upsilon}{\partial \xi}\right)^{2}-\frac{\partial^{2} \Upsilon}{\partial \xi^{2}} & \{\sin \gamma(\gamma-\eta)+ \\
& \left.+\frac{4 \sin \gamma}{\mathrm{f}(\eta)+\mathbf{a}}\left[\mathbf{a} \eta+\sin ^{n-1} \gamma\left(\mathrm{I}-(\mathrm{I}-\eta)^{n}\right)\right]+\frac{2(\mathrm{I}+\mathbf{a})}{\mathrm{f}(\eta)+\mathbf{a}} \phi m \sin ^{m} \gamma\right\},
\end{aligned}
$$

so that after an integration

$$
\begin{aligned}
\stackrel{(2)}{\tau}=k_{\mathrm{I}}(\xi, \tau)-\left(\frac{\partial \gamma}{\partial \xi}\right)^{2} \sin \gamma \eta & \\
& -\frac{\partial^{2} \Upsilon}{\partial \xi^{2}}\left\{\sin \gamma\left[\eta \Gamma-\frac{\eta^{2}}{2}-2\left(\mathrm{I}-(\mathrm{I}-\eta)^{2}\right)\right]+\mathfrak{A}\left(G_{n}(x)-G_{n}(x(\mathrm{I}-\eta))\right)\right\},
\end{aligned}
$$

is obtained, in which

$$
\begin{aligned}
x & =\sin \gamma / \mathfrak{a}^{1 /(n-1)}, \\
\mathfrak{A} & =4\left(\sin ^{n-1} \gamma \mathfrak{a}^{(2-n) /(n-1)}+\mathbf{a}^{\mathrm{I} /(n-1)}\right)+2(\mathrm{I}+\mathfrak{a}) \phi_{m} \sin ^{m-1} \gamma \mathfrak{a}^{(2-n) /(n-1)}, \\
G_{n}(x) & =\int_{0}^{x} \frac{d x^{\prime}}{x^{\prime n-1}+\mathrm{I}}= \begin{cases}\ln (x+1), & \text { for } n=2, \\
\tan ^{-1}(x), & \text { for } n=3 .\end{cases}
\end{aligned}
$$

The boundary condition for $\tau$ at $\eta=\Upsilon$ implies that

$$
\begin{aligned}
& \stackrel{(2)}{\tau(\eta}=\Gamma)=-\frac{\partial Y}{\partial \xi} \stackrel{(\mathrm{r})}{\left(\sigma_{x}-\sigma_{y}\right)} \\
& =-\frac{2(\mathrm{I}+\mathbf{a})}{\mathrm{f}(Y)+\mathrm{a}}\left(\frac{\partial \Upsilon}{\partial \xi}\right)^{2}\left\{\frac{2 \sin \gamma}{\mathrm{I}+\mathrm{a}}\left[\mathrm{a} Y+\sin ^{n-1} \gamma\left(\mathrm{r}-(\mathrm{I}-Y)^{n}\right)\right]+\phi m \sin ^{m} \gamma\right\} .
\end{aligned}
$$


Equations (A.ro) and (A.r2) together allow the determination of the constant of integration $k_{\mathrm{r}}(\xi, \tau)$. Once it is determined, $\tau$ may be written in the form

$$
\stackrel{(2)}{\tau}=-\stackrel{(2)}{T}_{11}(\eta, r)\left(\frac{\partial r}{\partial \xi}\right)^{2}-\stackrel{(2)}{T}_{12}(\eta, r) \frac{\partial^{2} r}{\partial \xi^{2}},
$$

in which

$$
\begin{aligned}
& \left.{\stackrel{(2)}{T_{\mathrm{II}}}}_{\eta}, Y\right)=\left\{\sin \gamma(\eta-Y)+\frac{4 \sin \gamma}{\mathrm{f}(Y)+\mathfrak{a}}\left[\mathfrak{a} Y+\sin ^{n-\mathrm{I}} \gamma\left(\mathrm{I}-(\mathrm{I}-Y)^{n}\right)\right]+\frac{2(\mathrm{I}+\mathfrak{a})}{\mathrm{f}(Y)+\mathfrak{a}} \phi m \sin ^{m} \gamma\right\}, \\
& \stackrel{(2)}{T}_{12}(\eta, Y)=\mathfrak{A}\left[G_{n}(x(\mathrm{I}-Y))-G_{n}(x)\right]+ \\
& +\sin \gamma\left\{\left[\left(\eta \gamma-\frac{\eta^{2}}{2}\right)-\left(\Upsilon^{2}-\frac{\Upsilon^{2}}{2}\right)\right]-2\left[\left(\mathrm{I}-(\mathrm{I}-\eta)^{2}\right)-\left(\mathrm{I}-(\mathrm{I}-\Upsilon)^{2}\right)\right]\right\} .
\end{aligned}
$$

Beyond this point calculations are very involved. For this reason we shall restrict ourselves henceforth to the necessary minimum. Substituting (A.13) and the fifth of Equations (25) into the differential equation for $u$,

$$
\frac{\partial u}{\partial \eta}=-\frac{\partial v}{\partial \xi}+\frac{2}{\mathrm{I}+\mathfrak{a}}\left(\mathbf{a}+n\left(\sin \gamma(\mathrm{I}-\eta)^{n-1}\right) \stackrel{(2)}{\tau}\right.
$$

and using the boundary condition

$$
\stackrel{(2)}{u_{\eta=0}}=-\phi m \sin ^{m-1} \gamma\left[\stackrel{(2)}{T}_{11}(0, r)\left(\frac{\partial \Upsilon}{\partial \xi}\right)^{2}+\stackrel{(2)}{T}_{12}(\mathrm{o}, \Upsilon) \frac{\partial^{2} \Upsilon}{\partial \xi^{2}}\right],
$$

it is straightforward to show that

$$
\stackrel{(2)}{u}=\stackrel{(2)}{U_{11}}(\eta, r) \frac{\partial^{2} Y}{\partial \xi^{2}}-\stackrel{(2)}{U_{12}}(\eta, r)\left(\frac{\partial \Upsilon}{\partial \xi}\right)^{2}
$$

Alternatively, the continuity equation

$$
\frac{\partial u}{\partial \xi}+\frac{\partial v}{\partial \eta}=0,
$$

determines $v$ as follows

$$
\stackrel{(2)}{v}=-\stackrel{(2)}{V_{11}}(\eta, r) \frac{\partial^{3} \Gamma}{\partial \xi^{3}}-\stackrel{(2)}{V_{12}}(\eta, r) \frac{\partial^{2} \Upsilon}{\partial \xi^{2}} \frac{\partial \Gamma}{\partial \xi}-\stackrel{(2)}{V_{13}}(\eta, Y)\left(\frac{\partial \Upsilon}{\partial \xi}\right)^{3}
$$

with

$$
\begin{aligned}
& \stackrel{(2)}{V}_{\mathrm{II}}(\eta, r)=\int_{0}^{\eta} \stackrel{(2)}{U}_{\mathrm{II}}\left(\eta^{\prime}, r\right) \mathrm{d} \eta^{\prime}, \\
& \stackrel{(2)}{V_{12}}(\eta, r)=\int_{0}^{\eta}\left\{\frac{\partial \stackrel{(2)}{U}_{\mathrm{II}}\left(\eta^{\prime}, r\right)}{\partial \Gamma}-2 \stackrel{(2)}{U}_{\mathrm{I} 2}\left(\eta^{\prime}, r\right)\right\} \mathrm{d} \eta^{\prime}, \\
& \stackrel{(2)}{V}_{13}(\eta, r)=-\int_{0}^{\eta} \frac{\partial(2)}{\partial U_{12}\left(\eta^{\prime}, r\right)} \frac{1}{\partial} \mathrm{d} \eta^{\prime} .
\end{aligned}
$$

These results now enable us to corroborate Equation (4.9). Indeed, with Equations (A.6), (A.7), (A.1 7), and (A.18) the surface-wave equation, the first of Equations (14), assumes the form of Equation (30), in which

$$
\begin{aligned}
& \text { 观 }(r):=-{\stackrel{(\mathrm{I})}{V_{11}}}_{(1}(r, r) \text {, } \\
& \mathfrak{E}(r):=\stackrel{(1)}{V}_{12}(r, \Upsilon)-\stackrel{(1)}{U}_{11}(r, \Upsilon), \\
& \mathfrak{f}(r):=-\stackrel{(2)}{V}_{\mathrm{II}}(r, r), \\
& \text { (6) }(r):=-\stackrel{(2)}{U}_{\mathrm{II}}(r, r)-\stackrel{(2)}{V_{12}}(r, r) \text {, } \\
& \text { 更 }(r):=\stackrel{(2)}{U}_{\mathrm{I2}}(r, r)-\stackrel{(2)}{V}_{\mathrm{I} 3}(r, r) \text {. }
\end{aligned}
$$

Evaluation of the above functions hinges on explicit expressions for the functions $\stackrel{(2)}{U}_{11}$ and $\stackrel{(2)}{U}_{12}$. Since in the main body of this article only 现 and $\mathcal{J}^{\mathbb{F}}$ need explicitly be known, it suffices to determine $U_{11}$. We leave it to the reader to prove that Equations (A.r 4$)-($ A.r 9 ) lead to the following expressions for $\mathfrak{J}$ (I): 


$$
\begin{aligned}
\mathbb{J}(\mathrm{I})=-\frac{2 \sin \gamma}{\mathrm{I}+\mathrm{a}}\left[\frac{\mathrm{a}}{24}+\frac{\sin ^{n-1} \gamma}{n+\mathrm{I}}\left(\frac{n+\mathrm{I}}{6}-\frac{\mathrm{I}}{2}+\frac{\mathrm{I}}{n+\mathrm{I}}-\frac{\mathrm{I}}{(n+2)(u+3)}\right)\right]+ \\
+\frac{2}{\mathrm{I}+\mathfrak{a}}\left[\mathbf{a} \boldsymbol{\tau}_{\mathrm{I} 2}(\mathrm{I})+\boldsymbol{\tau}_{\mathrm{I} 2}(\mathrm{I})\right]+\phi m \sin ^{m-1} \gamma\left[\frac{4}{3} \sin \gamma+\mathfrak{A}\left(G_{n}(\mathrm{o})-G_{n}(x)\right)\right],
\end{aligned}
$$

where

$$
\begin{aligned}
& \stackrel{\mathrm{I}}{\mathrm{I} 2}_{(\mathrm{I})}=\frac{3}{8} \sin \gamma+\mathfrak{A}\left\{\frac{\mathrm{I}}{2} G_{n}(\mathrm{o})-\frac{L_{n}(x)}{x}+\frac{\mathrm{I}}{x^{2}}\left(R_{n}(x)-R_{n}(\mathrm{o})\right)\right\}, \\
& { }^{2} \tau_{12}(\mathrm{I})=\sin ^{n-1} \gamma\left\{9 \sin \gamma\left[2\left(\frac{\mathrm{I}}{6}-\frac{\mathrm{I}}{2(n+\mathrm{I})}+\frac{\mathrm{I}}{(n+\mathrm{I})(n+2)}\right)+\frac{\mathrm{I}}{(n+\mathrm{I})(n+2)(n+3)}\right]+\right. \\
& \left.+\mathfrak{A}\left\langle\left(\mathrm{I}+\frac{\mathrm{I}}{n+\mathrm{I}}\right) G_{n}(\mathrm{o})-\frac{\mathrm{I}}{x^{n}}\left[O_{n}(x)-\frac{\mathrm{I}}{x}\left(P_{n}(x)-P_{n}(\mathrm{o})\right)\right]\right\rangle\right\}, \\
& L_{n}(x)= \begin{cases}(1+x) \ln (\mathrm{I}+x)-x, & n=2, \\
x \arctan x-\frac{1}{2} \ln \left(1+x^{2}\right), & n=3,\end{cases} \\
& R_{n}(x)= \begin{cases}\frac{1}{2}\left[(\mathrm{I}+x)^{2}\left(\ln (\mathrm{I}+x)-\frac{1}{2}\right)-x^{2}\right], & n=2, \\
\frac{1}{2}\left[\left(x^{2}-\mathrm{I}\right) \arctan x+x-x \ln \left(\mathrm{I}+x^{2}\right)\right], & n=3,\end{cases} \\
& O_{n}(x)= \begin{cases}\left(x^{2}-1\right) \ln (1+x)+x-\frac{1}{2} x^{2}, & n=2, \\
x^{3} \arctan x-\frac{1}{2} x^{2}+\frac{1}{2} \ln \left(1+x^{2}\right), & n=3,\end{cases} \\
& P_{n}(x)= \begin{cases}\frac{1}{3}\left[\left(x^{3}-3 x-2\right) \ln (1+x)-\frac{5}{6} x^{3}+2 x^{2}+2 x\right], & n=2, \\
\frac{1}{4}\left[\left(x^{4}+3\right) \arctan x+2 \ln \left(1+x^{2}\right)-x^{3}-3^{x}\right], & n=3 .\end{cases}
\end{aligned}
$$

\section{REFERENCES}

Barnes, P., and others. 1971. Friction and creep of polycrystalline ice, by P. Barnes, D. Tabor, and J. C. F. Walker. Proceedings of the Royal Society of London, Ser. A, Vol. 324, No. 1557, p. 127-55.

Benney, D. J. 1966. Long waves on liquid films. Journal of Mathematics and Physics, Vol. 45, No. 2, p. I50-55. Budd, W. F. I $970[\mathrm{a}]$. Ice flow over bedrock perturbations. Fournal of Glaciology, Vol. 9, No. 55, p. $29-48$.

Budd, W. F. 1970[b]. The longitudinal stress and strain-rate gradients in ice masses. Fournal of Glaciology, Vol. 9, No. 55, p. 19-27.

Budd, W. F. 1971. Stress variations with ice flow over undulations. Fournal of Glaciology, Vol. 10, No. 59, p. $177-95$.

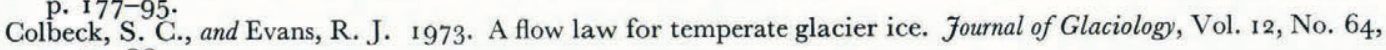
p. $71-86$.

Cole, J. D. I968. Perturbation methods in applied mathematics. Waltham, Mass., Blaisdell Publishing Co.

Frankenstein, G. E. 1968. Strength of ice sheets. Canada. National Research Council. Associate Committee on Geotechnical Research. Technical Memorandum No. 92, p. 79-87.

Hutter, K. In press. The effect of longitudinal strain on the shear stress of an ice sheet. In defence of using stretched coordinates. Fournal of Glaciology, Vol. 27, No. 95.

Hutter, K., and others. In press. First-order stresses and deformations in glaciers and ice sheets, by K. Hutter, F. Legerer, and U. Spring. Journal of Glaciology, Vol. 27, No. 96.

Jellinek, H. H. G., and Brill, R. 1956. Viscoelastic properties of ice. Journal of Applied Physics, Vol. 27, No. 10, p. $1198-209$.

Leibowich, S., and Seebass, R. A. 1974. Nonlinear waves. Ithaca and London, Cornell University Press.

Lick, W. 1970. The propagation of disturbances on glaciers. Journal of Geophysical Research, Vol. 75, No. 12, p. $2189-97$.

Lliboutry, L. A. 1969. The dynamics of temperate glaciers from the detailed viewpoint. Fournal of Glaciology, Vol. 8, No. 53 , p. $185^{-205}$.

Nayfeh, A. H. 1973. Perturbation methods. New York, etc., John Wiley and Sons, Inc.

Nye, J. F. 1960. The response of glaciers and ice-sheets to seasonal and climatic changes. Proceedings of the Royal Society of London, Ser. A, Vol. 256, No. 1287, p. 559-84.

Nye, J. F. ${ }_{1963}$ [a]. On the theory of the advance and retreat of glaciers. Geophysical fournal of the Royal Astronomical Society, Vol. 7 , No. 4 , p. $431-56$.

Nye, J. F. $\operatorname{l~}_{96}[$ [b]. The response of a glacier to changes in the rate of nourishment and wastage. Proceedings of the Royal Society of London, Ser. A, Vol. 275, No. 1360, p. 87-1 I2.

Ramseier, R. O., and Dickins, D. F. 1972. A new approach to field and laboratory tests of tensile, compressive, and flexural strength of polycrystalline, fresh water ice. (In [International Association of Hydraulic Research.] I.A.H.R. symposium. Ice and its action on hydraulic structures, Leningrad, USSR, 26-29 September 1972. [Leningrad, International Association of Hydraulic Research], [Vol. 1], p. 143-47.)

Stoker, J. J. 1957. Water waves, the mathematical theory with applications. New York, Interscience Publishers, Inc.

Thompson, D. E. 1979. Stability of glaciers and ice sheets against flow perturbations. Fournal of Glaciology, Vol. 24, No. 9o, p. 427-4I.

Van Dyke, M. I975. Perturbation methods in fluid mechanics. Stanford, California, Parabolic Press. 